\title{
Ocorrência de leishmaniose em gatos de área endêmica para leishmaniose visceral
}

\author{
Occurrence of leishmaniasis in cats from endemic area for visceral leishmaniasis
}

\author{
Thiago André Carreo COSTA ${ }^{1}$; Claudio Nazaretian ROSSI ${ }^{2}$; Márcia Dalastra LAURENTI ${ }^{3}$; Ana Amélia \\ Domingues GOMES ${ }^{4}$; Juliana Peloi VIDES ${ }^{5}$ Ludmila Silva Vicente SOBRINHO ${ }^{5}$; \\ Mary MARCONDES
}

\begin{abstract}
${ }^{1}$ Curso de Medicina Veterinária da Faculdade Pio Décimo, Aracaju-SE ${ }^{2}$ Faculdade de Medicina Veterinária e Zootecnia da Universidade de São Paulo, São Paulo-SP ${ }^{3}$ Departamento de Patologia da Faculdade de Medicina da Universidade de São Paulo, São Paulo-SP ${ }^{4}$ Faculdade de Ciências Agrárias e Veterinárias da Universidade Estadual Paulista, Jaboticabal-SP ${ }^{5}$ Curso de Medicina Veterinária da Universidade Estadual Paulista, Araçatuba-SP
\end{abstract}

\begin{abstract}
Resumo
Apesar da descrição de vários casos de leishmaniose felina no mundo, pouco se sabe sobre a importância do gato como um reservatório da doença. $\mathrm{O}$ objetivo do presente estudo foi determinar a ocorrência de leishmaniose em gatos provenientes de uma área endêmica para leishmaniose visceral no Brasil. Duzentos gatos foram incluídos no estudo. A presença de infecção foi determinada por meio do achado de formas amastigotas do parasita em esfregaços obtidos por punção biópsia aspirativa de linfonodos, medula óssea, baço e fígado, e pela presença de anticorpos anti-L. chagasi pelo teste de ELISA indireto. Nossos resultados demonstraram uma prevalência de infecção em 14,5\% (31/200) dos gatos na população estudada, sendo 4\% (8/200) por diagnóstico parasitológico e 11,5\% (23/200) por sorologia.
\end{abstract}

Palavras-chave: ELISA. Imunodiagnóstico. Felinos. Leishmania sp.

\begin{abstract}
Despite the description of several cases of feline leishmaniasis around the world, little information is available about
the importance of the cat as a reservoir of the disease. The aim of the present study was to determine the occurrence
of leishmaniasis in cats from an endemic area for visceral leishmaniasis in Brazil. Two hundred cats were included in
this study. Infection was evaluated through the presence of amastigotes in stained smears from fine-needle aspirates
of lymph nodes, bone marrow, spleen and liver, and by antibody reactivity against Leishmania chagasi using indirect
ELISA. Our results showed a prevalence of infection in 14.5\% (31/200) of the feline population studied, with $4 \%(8 / 200)$
Abstract
Despite the description of several cases of feline leishmaniasis around the world, little information is available about
the importance of the cat as a reservoir of the disease. The aim of the present study was to determine the occurrence
of leishmaniasis in cats from an endemic area for visceral leishmaniasis in Brazil. Two hundred cats were included in
this study. Infection was evaluated through the presence of amastigotes in stained smears from fine-needle aspirates
of lymph nodes, bone marrow, spleen and liver, and by antibody reactivity against Leishmania chagasi using indirect
ELISA. Our results showed a prevalence of infection in 14.5\% (31/200) of the feline population studied, with $4 \%(8 / 200)$

Abstract
Despite the description of several cases of feline leishmaniasis around the world, little information is available about
the importance of the cat as a reservoir of the disease. The aim of the present study was to determine the occurrence
of leishmaniasis in cats from an endemic area for visceral leishmaniasis in Brazil. Two hundred cats were included in
this study. Infection was evaluated through the presence of amastigotes in stained smears from fine-needle aspirates
of lymph nodes, bone marrow, spleen and liver, and by antibody reactivity against Leishmania chagasi using indirect
ELISA. Our results showed a prevalence of infection in 14.5\% (31/200) of the feline population studied, with $4 \%(8 / 200)$

Abstract
Despite the description of several cases of feline leishmaniasis around the world, little information is available about
the importance of the cat as a reservoir of the disease. The aim of the present study was to determine the occurrence
of leishmaniasis in cats from an endemic area for visceral leishmaniasis in Brazil. Two hundred cats were included in
this study. Infection was evaluated through the presence of amastigotes in stained smears from fine-needle aspirates
of lymph nodes, bone marrow, spleen and liver, and by antibody reactivity against Leishmania chagasi using indirect
ELISA. Our results showed a prevalence of infection in 14.5\% (31/200) of the feline population studied, with $4 \%(8 / 200)$

Abstract
Despite the description of several cases of feline leishmaniasis around the world, little information is available about
the importance of the cat as a reservoir of the disease. The aim of the present study was to determine the occurrence
of leishmaniasis in cats from an endemic area for visceral leishmaniasis in Brazil. Two hundred cats were included in
this study. Infection was evaluated through the presence of amastigotes in stained smears from fine-needle aspirates
of lymph nodes, bone marrow, spleen and liver, and by antibody reactivity against Leishmania chagasi using indirect
ELISA. Our results showed a prevalence of infection in 14.5\% (31/200) of the feline population studied, with $4 \%(8 / 200)$

Abstract
Despite the description of several cases of feline leishmaniasis around the world, little information is available about
the importance of the cat as a reservoir of the disease. The aim of the present study was to determine the occurrence
of leishmaniasis in cats from an endemic area for visceral leishmaniasis in Brazil. Two hundred cats were included in
this study. Infection was evaluated through the presence of amastigotes in stained smears from fine-needle aspirates
of lymph nodes, bone marrow, spleen and liver, and by antibody reactivity against Leishmania chagasi using indirect
ELISA. Our results showed a prevalence of infection in 14.5\% (31/200) of the feline population studied, with $4 \%(8 / 200)$

Abstract
Despite the description of several cases of feline leishmaniasis around the world, little information is available about
the importance of the cat as a reservoir of the disease. The aim of the present study was to determine the occurrence
of leishmaniasis in cats from an endemic area for visceral leishmaniasis in Brazil. Two hundred cats were included in
this study. Infection was evaluated through the presence of amastigotes in stained smears from fine-needle aspirates
of lymph nodes, bone marrow, spleen and liver, and by antibody reactivity against Leishmania chagasi using indirect
ELISA. Our results showed a prevalence of infection in 14.5\% (31/200) of the feline population studied, with $4 \%(8 / 200)$ of positivity by parasitological diagnosis and $11.5 \%(23 / 200)$ by serology.
\end{abstract}

Keywords: ELISA. Immunodiagnosis. Feline. Leishmania sp.

\section{Introdução}

A infecção natural de um gato doméstico por Leishmania sp. teve sua primeira descrição em 1912, na Argélia, em um animal de quatro meses de idade, que convivia com um cão e uma criança, portadores de leishmaniose visceral. O diagnóstico baseou-se no achado de formas amastigotas do parasito em medula óssea, sem a identificação da espécie causadora de enfermidade ${ }^{1}$. Da descrição do primeiro caso clínico até os dias de hoje, a literatura mundial tem registros de $45 \mathrm{ca}$ sos positivos pelo exame parasitológico para Leishmania sp. em diversos países como nos Estados Unidos², França $^{3,4}$, Espanha ${ }^{5,6,7}$, Itáliaa ${ }^{8,910}$, Portugal ${ }^{11,12}$, Suíça ${ }^{13}$ e Brasil $^{14,15,16,17,18,19,20}$. Destes casos, 24 (53,3\%) ocorreram no Novo Mundo e 21 (46,6\%) no Velho Mundo.

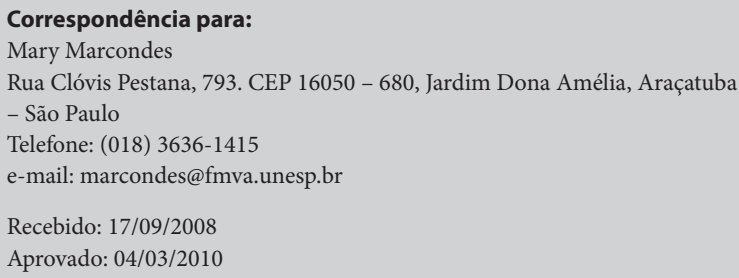

Aprovado: 04/03/2010 
Apesar da ocorrência de infecções esporádicas, os felinos não são considerados, até o momento, um reservatório importante da doença e existem discordâncias na literatura com relação à susceptibilidade dos felídeos domésticos à infecção por Leishmania sp. ${ }^{10,21,22}$. Acredita-se que gatos infectados possuam certo grau de resistência natural à enfermidade provavelmente relacionada a fatores genéticos ${ }^{10,23,24}$.

O crescente aumento na prevalência de leishmaniose visceral canina no Brasil, particularmente no Estado de São Paulo, área considerada indene até o relato do primeiro caso no ano de 1998, vem gerando preocupações em profissionais que atuam junto a órgãos de saúde pública, em clínicos veterinários de pequenos animais e também na população residente em áreas de risco. Nesse contexto, surge a necessidade de identificar possíveis reservatórios domésticos, entre eles os felinos, conforme salientado por Simões-Mattos ${ }^{22}$. Apesar da existência de alguns estudos pesquisando a soroprevalência da infecção em populações de felinos residentes em áreas endêmicas, não está claro ainda se as baixas prevalências de infecção e de doença, em gatos provenientes de áreas endêmicas, são devidas a falhas na detecção de anticorpos ou ao fato dos gatos apresentarem resistência natural à leishmaniose $\mathrm{e}^{7,8,12,20,25,26}$.

Baseando-se na hipótese de que gatos podem ser infectados por Leishmania chagasi, o presente estudo teve como objetivo determinar a soropositividade de infecção por Leishmania sp. em 200 gatos provenientes de área endêmica para leishmaniose visceral por meio da técnica de ELISA indireto.

\section{Material e Método}

Para a realização do presente estudo foram colhidas amostras de sangue de dois grupos de animais. $\mathrm{O}$ primeiro grupo foi constituído por 200 gatos adultos, independente de sexo ou raça, provenientes do Centro de Controle de Zoonozes (CCZ) do município de Araçatuba, São Paulo, Brasil, área endêmica para leishmaniose visceral; o segundo foi formado por 53 gatos residentes no município de Santos, São Paulo, área não endêmica para a doença; utilizados como grupo controle. Nos gatos do primeiro grupo foram realizadas citologias aspirativas por agulha fina de linfonodos poplíteos, medula óssea, baço e fígado para a pesquisa de formas amastigotas de Leishmania sp., por meio de exame parasitológico direto, em lâminas coradas com corante hematológico e observadas ao microscópio óptico, em objetiva de 100x (imersão).

Após a titulação em bloco, padronizaram-se as seguintes condições para o teste de ELISA indireto: as microplacas foram cobertas com antígeno total de Leishmania chagasi, numa concentração de $10 \mu \mathrm{g} / \mathrm{mL}$ em tampão carbonato $0,05 \mathrm{M}, \mathrm{pH}$ 9,6, e incubadas por 18 horas a 4 ${ }^{\circ} \mathrm{C}$. As placas foram bloqueadas com $200 \mu \mathrm{L}$ de solução salina tamponada acrescida de leite em pó desnatado a $10 \%$, e incubadas a $37^{\circ} \mathrm{C}$ durante duas horas em câmara úmida. Adicionaram-se $100 \mu \mathrm{L}$ por poço das amostras de soros dos animais controle positivo e controle negativo, e dos gatos dos grupos experimentais, diluídas 1:400 em PBS-T e soro fetal bovino a $10 \%$, e as placas foram incubadas por uma hora à temperatura ambiente. Colocaram-se $100 \mu \mathrm{L}$ por poço do conjugado anti-IgG total de gato ligado à peroxidase (A20-120P Bethyl, Montgomery, USA), na diluição de 1:40000 em PBS-T. Após 45 minutos de incubação a $37^{\circ} \mathrm{C}$ foram adicionados $100 \mu \mathrm{L}$ da solução de tetrametilbenzidina dihidroclorada (TMB) (Código 55214, BD Biosciences Pharmingen, San Diego, USA) com posterior incubação da placa por $30 \mathrm{minu-}$ tos, ao abrigo de luz, em temperatura ambiente. A reação foi interrompida adicionando-se a cada poço $50 \mu \mathrm{L}$ de ácido sulfúrico $0,5 \mathrm{~N}$ e a densidade óptica (D.O.) foi determinada em leitor de ELISA (Labsystems Multiskan EX - Thermo Fisher Scientific Inc. - Waltham, MA), utilizando-se filtro de $450 \mathrm{~nm}$. Entre cada etapa as placas foram lavadas com solução salina tamponada contendo 0,05\% de Twen 20 (PBS-T), por quatro vezes. Os resultados foram expressos pela média da densidade óptica obtida dos soros em duplicata. Como controle positivo da reação utilizou-se soro de um gato parasitologicamente 
positivo com elevados títulos de IgG anti-Leishmania sp. proveniente do município de Araçatuba, São Paulo, e como controle negativo, soro de um gato proveniente de área não endêmica para a doença. A determinação do ponto de corte $(0,365)$ foi realizada com as 53 amostras de soros dos gatos provenientes de área não endêmica. A sensibilidade e a especificidade do método de ELISA indireto foram determinadas considerando-se como referência os resultados obtidos no exame parasitológico direto e o coeficiente Kappa foi usado para avaliar a concordância entre os dois métodos. A interpretação da concordância foi feita de acordo com Landis e $\mathrm{Koch}^{27}$. As análises foram realizadas utilizando-se o programa "Statistical Analysis System" (SAS ${ }^{\circledR}$ Learning Edition 2.0 - SAS Institute Inc., Cary, NC, EUA. 2005).

\section{Resultados}

Dos 200 gatos avaliados pelo exame parasitológico direto, em oito (4\%) foram evidenciadas formas amastigotas de Leishmania sp. (Tabela 1). Quatro gatos apresentaram formas amastigotas de Leishmania sp. somente em citologia de linfonodos poplíteos. Em apenas um animal observou-se o parasito na punção biópsia aspirativa de fígado, sendo negativo nos demais tecidos avaliados. Já em um felino evidenciou-se Leishmania sp. tanto no linfonodo poplíteo quanto na medula óssea, diferente de outros dois, em que foram identificadas formas amastigotas em preparados citológicos de linfonodos poplíteos, baço e medula óssea. Vinte e três gatos (11,5\%) foram sorologicamente positivos pela técnica de ELISA indireto. Dos oito animais com diagnóstico positivo ao exame parasitológico direto, apenas dois (25\%) foram positivos também pela técnica de ELISA indireto. A concordância entre os dois testes foi considerada fraca (Tabela 2). Os valores de sensibilidade e especificidade para o método de ELISA indireto foram 25\% e $89 \%$, respectivamente. Dos animais positivos, apenas dois (25\%) apresentavam alterações ao exame físico, caracterizadas por lesões dermatológicas crostosas na região cervical dorsal, acompanhadas de hepatoesplenomegalia.

\section{Discussão e Conclusões}

Apesar de dois gatos soropositivos apresentarem lesões de pele e hepatoesplenomegalia, não é possível

Tabela 1 - Valores absolutos e percentuais observados para a presença de formas amastigotas de Leishmania sp. em amostras de punção biópsia aspirativa (PBA) de linfonodos poplíteos, baço, fígado e medula óssea de 200 gatos provenientes de área endêmica para leishmaniose visceral - Araçatuba - SP - 2008

\begin{tabular}{ccc}
\hline Tecidos & \multicolumn{2}{c}{ PBA } \\
\cline { 2 - 3 } & Número de Animais & Percentagem (\%) \\
\hline Linfonodos poplíteos & 7 & 87,5 \\
Medula óssea & 3 & 37,5 \\
Baço & 2 & 25,0 \\
Fígado & 1 & 12,5 \\
\hline Total & 8 & 100 \\
\hline
\end{tabular}

Tabela 2 - Resultados da pesquisa de Leishmania sp por meio de exame parasitológico direto e de sorologia pela técnica de ELISA indireto em amostras de 200 gatos de área endêmica para leishmaniose visceral - Araçatuba - SP - 2008

\begin{tabular}{ccccccc}
\hline & & \multicolumn{2}{c}{ Parasitológico } & \multirow{2}{*}{ Kappa } & Concordância $^{*}$ \\
\cline { 2 - 4 } & & Positivo & Negativo & & \\
\hline \multirow{2}{*}{ ELISA } & Positivo & 2 & 21 & \multirow{2}{*}{0,0894} & Fraca \\
\cline { 2 - 4 } & Negativo & 6 & 171 & & \\
\hline
\end{tabular}

${ }^{\star}$ Landis e $\mathrm{Koch}^{27}$. 
afirmar que tais alterações sejam decorrentes de uma infecção por Leishmania sp., uma vez que não foram excluídas outras enfermidades infecciosas nos animais avaliados. Dos órgãos utilizados para a realização do exame parasitológico direto, os linfonodos poplíteos foram os que apresentaram maior positividade em um maior número de animais, sugerindo que deva ser a primeira opção quando da escolha do local onde realizar a punção biópsia aspirativa. A punção de medula óssea não elevou a sensibilidade do exame parasitológico, uma vez que os animais em que se observaram formas amastigotas na medula óssea também possuíam parasitas nos linfonodos poplíteos. Embora tenham sido encontrados parasitas no fígado de apenas um gato, a punção deste órgão demonstrou ser importante, uma vez que no referido animal não foram observadas formas amastigotas de Leishmania sp em nenhum outro órgão linfoide.

A soropositividade em $11,5 \%$ dos gatos do presente estudo foi inferior à observada por Pennisi, Masucci e Catarsini ${ }^{25}$, ao avaliarem gatos sorologicamente positivos para imunodeficiência viral felina (FIV) na Itália, e Martín-Sánchez et al. 7 , em um estudo com 183 gatos encaminhados a clínicas veterinárias do sudeste da Espanha, que encontraram valores próximos a $60 \%$. Por outro lado, está relativamente próxima aos resultados obtidos por Solano-Gallego et al. ${ }^{26}$, ao determinarem a soroprevalência de leishmaniose visceral em 445 gatos provenientes da região noroeste da Bacia do Mediterrâneo e identificarem um valor de $5,25 \%{ }^{26}$, e superior aos resultados de Poli et al. ${ }^{8}$, que encontraram $0,9 \%$ de soropositividade em uma população de gatos da Toscana.

A técnica de ELISA apresentou baixa sensibilidade (25\%) quando comparada à observada em ensaios ca-

\section{Referências}

1.SERGENT, E.; SERGENT, E.; LOMBAARD, J.; QUILICHINI, M. La Leishmaniose à Alger. Infection simultanée d'um enfant, d'um chien et d'um chat dans la meme habitation. Bulletin de Societé de Pathologie Exotique, v. 5, p. 93-98, 1912.

2.BARNES, J. C.; STANLEY, O.; CRAIG, T. M. Diffuse cutaneous leishmaniasis in a cat. Journal of the American Veterinary Medical Association, v. 202, p. 416-418, 1993. ninos utilizando a mesma técnica, que variou de 94 a $99,5 \%{ }^{28,29,30}$. Um fator que poderia justificar este valor seria a diluição utilizada no soro e no conjugado ${ }^{26}$. No entanto, foram realizadas padronizações com diversas diluições de soro e de conjugado, de modo que este não pode ser considerado um fator determinante da sensibilidade da técnica.

No que diz respeito à especificidade (89\%), esta corrobora os valores descritos por Mancianti et al. ${ }^{28}$, Laurenti et al..$^{29}$ e Zanette ${ }^{30}$, que variaram de $84,4 \%$ a $100 \%$ em estudos caninos. A concordância observada entre $o$ diagnóstico parasitológico e a técnica de ELISA indireto foi considerada fraca, baseando-se no coeficiente Kappa. Chama a atenção o fato de que dos oito gatos com exame parasitológico positivo, apenas dois eram sorologicamente positivos. Esta observação concorda com os relatos de Martín-Sánchez et al. ${ }^{7}$, que avaliaram amostras de soro de 183 gatos por imunofluorescência indireta e verificaram que os animais com os mais altos títulos de anticorpos foram os que apresentaram a menor proporção de positividade para a presença de DNA na técnica de PCR. Por outro lado, as maiores proporções de PCR positivos ocorreram em gatos com baixos títulos de anticorpos. Estes resultados sugerem que a resposta imune à infecção por Leishmania sp em gatos difere da observada em cães, o que deve explicar o pequeno número de animais infectados e sintomáticos. Esse fato pode subestimar o número real de gatos infectados, desta forma, facilitando a transmissão dos parasitos, como salientado por Portús et al. ${ }^{31}$. Na população de gatos estudada no presente experimento observou-se uma prevalência de $14,5 \%$ de positividade para leishmaniose, sendo $4 \%$ por exame parasitológico direto e $11,5 \%$ por sorologia.

3.OZON, C.; MARTY, P.; PRATLONG, F.; BRETON, C.; BLEIN, M.; LELIẼVRE, A.; HAAS, P. Disseminated feline leishmaniosis due to Leishmania infantum in Southern France. Veterinary Parasitology, v. 75, n. 2-3, p. 273-277, 1998.

4.GREVOT, A.; JAUSSAUD HUGUES, P.; MARTY, P.; PRATLONG, F.; OZON, C.; HAAS, P.; BRETON, C.; BOURDOISEAU, G. Leishmaniosis due to Leishmania 
infantum in a FIV and FelV positive cat with a squamous cell carcinoma diagnosed with histological, serological and isoenzymatic methods. Parasite, v. 12, n. 3, p. 271-275, 2005

5.HERVAS, J.; CHACON-MANRIQUE DE LARA, F.; SANCHEZISARRIA, M. A.; PELLICER, S.; CARRASCO, L.; CASTILLO, J. A.; GOMEZ-VILLAMANDOS, J. C. Two cases of feline visceral and cutaneous leishmaniosis in Spain. Journal of Feline Medicine and Surgery, v. 1, n. 2, p. 101-105, 1999.

6.LEIVA, M.; LORET, A.; PEÑA, T.; ROURA, X. Therapy of ocular and visceral leishmaniasis in a cat. Veterinary Ophthalmology, v. 8, n. 1, p. 71-75, 2005.

7.MARTÍN-SÁNCHEZ, J.; ACEDO, C.; MUÑOS-PÉREZ, M.; PESSON, B.; MARCHAL, O.; MORILLAS-MÁRQUEZ, F Infection by Leishmania infantum in cats: epidemiological study in Spain. Veterinary Parasitology, v. 30, n. 145, p. 267-273, 2007.

8.POLI, A.; ABRAMO, F; BARSOTTI, P.; LEVA, S.; GRAMICCIA, M.; LUDOVISI, A.; MANCIANTI, F. Feline leishmaniosis due to Leishmania infantum in Italy. Veterinary Parasitology, v. 26, n. 106, p. 181-191, 2002.

9.PENNISI, M. G.; VENZA, M.; REALE, S.; VITALE, F.; GIUDICE, S. Case report of Leishmaniasis in four cats. Veterinary Research Communications, v. 28, p. 363-366, 2004. Supplement, 1.

10.VITA, S.; AGUZZI, I.; PETROTTA, E.; LUCIANI, A. Feline leishmanisis and erlichiosis: serological investigation in Abruzzo region. Veterinary Research Communications, v. 29, p. 319-321, 2005. Supplement, 2

11.COSTA DURÃO, J. F.; REBELO, E.; PELETEIRO, M. C.; CORREIA, J. J.; SIMÕES, G. Primeiro caso de leishmaniose em gato doméstico (Felis catus domesticus) detectado em Portugal (Concelho de Sesimbra). Nota preliminar. Revista Portuguesa de Ciências Veterinárias, v. 89, n. 511, p. 140-144, 1994.

12. MAIA, C.; NUNES, M.; CAMPINO, L. Importance of cats in zoonotic leishmaniasis in Portugal. Vector-Borne and Zoonotic Diseases, v. 8, p. 555-559, 2008.

13. RUFENACHT, S.; SAGER, H.; MULLER, N.; SCHAERER, V.; HEIER, A.; WELLE, M. M.; ROOSIE, P. J. Two cases of feline leishmaniasis in Switzerland. Veterinary Record, v. 156, p. 542-545, 2005.

14. MELLO, G. B. Verificação da infecção natural do gato (Felix domesticus) por um protozoário do gênero Leishmania. Brasil Médico, v. 54, n. 12, p. 180, 1940.

15.SAVANI, E. S. M. M.; CAMARGO, M. C. G. O; CARVALHO, M. R.; ZAMPIERI, R. A.; SANTOS, M. G.; D'ÁURIA, S. R. N.; SHAW, J. J.; FLOETER-WINTER, L. M. The first record in the Americas of an autochthonous case of Leishmania (Leishmania) infantum chagasi in a domestic cat (Felix catus) from Cotia County, São Paulo State, Brazil. Short communication. Veterinary Parasitology, v. 120, n. 3, p. 229 233,2004

16.DA SILVA, A. V.; DE SOUZA CÂNDIDO, C. D.; DE PITA PEREIRA, D.; BRAZIL, R. P.; CARREIRA, J. C. The first record of american visceral leishmaniasis in domestic cats from Rio de Janeiro, Brazil. Acta Tropica, v. 105, n. 1, p. 92-94, 2008.

17.PASSOS, V. M. A.; LASMAR, E. B.; GONTIJO, C. M. F.; FERNANDES, O.; DEGRAVE, W. Natural infection of a domestic cat (Felis domesticus) with Leishmania (Viannia) in the Metropolitan Region of Belo Horizonte, State of Minas Gerais, Brazil. Memórias do Instituto Oswaldo Cruz, v. 91, n. 1, p. $19-20,1996$.

18.SCHUBACH, A.; FIGUEIREDO, F. B.; PEREIRA, S. A.; MADEIRA, M. F.; SANTOS, I. B.; ANDRADE, M. V.; CUZZI, T.; MARZOCHI, M. C. A.; SCHUBACH, A. Americam cutaneous leishmaniasis in two cats from Rio de Janeiro, Brazil: first report of natural infection with Leishmania (Viannia) brasiliensis.Transactions of the Royal Soceity of Tropical Medicine and Hygiene, v. 98, p. 165-167, 2004.

19.DE SOUZA, A. L.; BARROS, E. M.; ISHIKAWA, E.; ILHA, I. M.; MARIN, G. R.; NUNES, V. L. Feline leishmaniasis due to Leishmania (Leishmania) amazonensis in Mato Grosso do Sul State, Brazil. Veterinary Parasitology, v. 128, n. 1-2, p. 41-45, 2005.

20.ROSSI, C. N. Ocorrência de Leishmania sp. em gatos do município de Araçatuba - São Paulo - Brasil. 2007. Dissertação (Mestrado) - Universidade Estadual Paulista, Faculdade de Ciências Agrárias e Veterinárias, Jaboticabal, 2007. p. 1-48.

21.KIRKPATRICCK, C. E.; FARRELL, J. P.; GOLDSCHMIDT, M. H. Leishmania chagasi and L. donovani: experimental infections in domestic cats. Experimental Parasitology, v. 58, n. 2, p. 125-131, 1984.

22.SIMÕES-MATTOS, L. O gato doméstico (Felis catus) como potencial hospedeiro rervatório de Leishamania (Viannia) braziliensis. 2005. 231 f. Tese (Doutorado) - Universidade Estadual do Ceará, Faculdade de Veterinária, 2005.

23.PENNISI, M. G. A high prevalence of feline leishmaniasis in southern Italy. In: KILLICK-KENDRICK, R. (Ed.). Canine Leishmaniasis: moving towards a solution. Boxmeer: Intervet International, 2002. p. 39-48.

24.MANCIANTI, F. Feline leishmaniasis: what's the epidemiological role of the cat? Parassitologia, v. 46, n. 1-2, p. 203-206, 2004

25.PENNISI, M. G.; MASUCCI, M.; CATARSINI, O. Presenza di anticorpi anti-Leishmania in gatti FIV+ che vivono in zona endemica. Atti della Società Italiana delle Scienze Veterinaire, v. 52, p. 265-266, 1998.

26.SOLANO-GALLEGO, L.; RODRÍGUEZ-CORTÉS, A. INIESTA, L.; QUINTANA, J.; PASTOR, J.; ESPADA, Y. PORTÚS, M.; ALBEROLA, J. Cross-sectional serosurvey of feline leishmaniasis in ecoregions around the Northwestern Mediterranean. American Journal of Tropical Medicine and Hygiene, v. 76, n. 4, p. 676-680, 2007.

27.LANDIS, J. R.; KOCH, G. G. The measurement of observer agreement for categorical data. Biometrics, v. 33, p. 159-174, 1977.

28. MANCIANTI, F.; FALCONE, M. L.; GIANNELLI, C.; POLI, A. Comparison between an enzyme-linked immunosorbent assay using a detergent-soluble Leishmania infantum antigen and indirect immunofluorescence for the diagnosis of canine leishmaniosis. Veterinary Parasitology, v. 59, n. 1, p. 13-21, 1995.

29.LAURENTI, M. D.; LEMOS, E. M.; REIS, A. B.; MOREIRA, M. A. B.; LUVIZOTTO, M. C. R.; CORBETT, C. E. P.; DIETZE, R. Evaluation of Kalazar detect rapid test for serodiagnosis of canine visceral leishmaniasis in Brazil. In: WORLD CONGRESS ON LEISHMANIASIS, 3., 2005. Italy. Abstract... Italy. 2005. p. 160.

30.ZANETTE, M. F. Comparação entre os métodos de ELISA, imunofluorescência indireta e imunocromatografia para o diagnóstico da leishmaniose visceral canina. 2006. $92 \mathrm{f}$. Dissertação (Mestrado em Ciência Animal) - Faculdade de Odontologia, Curso de Medicina Veterinária, Universidade Estadual Paulista, Araçatuba, 2006.

31.PORTÚS, M.; GÁLLEGO, M.; RIERA, C.; AISA, M.; FISA, R.; CASTILLEJO, S. Wild and domestic mammals in the life cycle of Leishmania infantum in Southwest Europe. A literature review and studies performed in Catalonia (Spain). Revista Iberica de Parasitologia, v. 62, p. 72, 2002. 